\title{
ANÁLISE DO DESEMPENHO FÍSICO E DO EQUILÍBRIO SOB INFLUÊNCIA DA CRIOTERAPIA EM ATLETAS DE FUTSAL
}

\author{
PHYSICAL PERFORMANCE AND BALANCE ANALYSIS UNDER INFLUENCE OF CRYOTHERAPY \\ IN INDOOR SOCCER ATHLETES
}

Original Article

ARTIGO ORIGINAL

Artículo Original

\author{
ANÁLISIS DEL DESEMPEÑO FÍSICO Y EQUILÍBRIO SOB INFLUENCIA DE CRIOTERAPIA \\ EN JUGADORES DE FÚTBOL SALA
}

\begin{abstract}
Thiago Rocha Freire' (Fisioterapeuta) Mylena Maria Salgueiro Santana' (Fisioterapeuta)

Jader Pereira de Farias Neto ${ }^{1}$ (Fisioterapeuta)

Marzo Edir da Silva Grigoletto' (Profissional de Educação Física) Walderi Monteiro da Silva Júnior ${ }^{1}$ (Fisioterapeuta)

1. Universidade Federal de Sergipe, Aracaju, SE, Brasil.

\section{Correspondência:}

Mylena Maria Salgueiro Santana.

Rua Maruim, 801, Centro.

Aracaju, SE, Brasil. 49010-160.

ftmylenasantana@gmail.com
\end{abstract}

\section{RESUMO}

Introdução: Crioterapia é a aplicação de modalidades de frio com temperatura de $0^{\circ} \mathrm{C}$ a $18,3^{\circ} \mathrm{C}$, podendo interferir no desempenho físico e equilíbrio, dependendo da capacidade do indivíduo em manter a estabilização, direcionar padrões de movimentos, controlar a postura e posição articular. Objetivo: Avaliar o desempenho físico, a frequência cardíaca e o equilíbrio estático com olhos abertos, em atletas de futsal (futebol de salão), antes e depois da crioimersão nos membros inferiores. Métodos: Trinta e dois indivíduos do gênero masculino participaram do estudo, distribuídos aleatoriamente em dois grupos: (A) grupo controle, imersão em água a $24^{\circ} \mathrm{C}$ por $10 \mathrm{~min}$; (B) grupo intervenção, crioimersão em água com gelo a $10^{\circ} \mathrm{C}$ por $10 \mathrm{~min}$. Os voluntários realizavam a avaliação do equilíbrio no baropodômetro, em seguida corriam em linha reta e em ziguezague por 100 m, entravam na crioimersão, terminando com uma nova avaliação. Foram analisados o desempenho físico, através dos tempos das corridas, a frequência cardíaca, por meio de um frequencímetro e o equilíbrio, através da baropodometria e estabilometria em apoio bipodal de olhos abertos. Resultados: O desempenho físico foi alterado após a crioimersão quando das análises intra e intergrupos. A frequência cardíaca apresentou diferença ao comparar-se antes e após a crio, porém sem diferenças em comparação ao controle. O equilíbrio não foi alterado após a crioimersão e nem em comparação com o grupo controle. Conclusão: A crioimersão prejudicou o desempenho físico, quando a atividade foi imediatamente após a mesma, sendo desta forma o gelo não aconselhável quando se deseja desempenho na atividade desportiva. Porém não apresentou interferência na frequência cardíaca e no equilíbrio de atletas de futsal, que podem não ter sido alterados devido ao tempo da crioimersão.

Palavras-chave: avaliação, hipotermia induzida, desempenho atlético, equilíbrio postural.

\section{ABSTRACT}

Introduction: Cryotherapy is the application of methods of cold temperature of $0^{\circ} \mathrm{C}$ to $18.3^{\circ} \mathrm{C}$, which may interfere in physical performance and balance, depending on the individual's capacity of maintaining stabilization, targeting movement patterns, controlling posture and joint position. Objective: To evaluate physical performance, heart rate and static balance with eyes open, in indoor soccer players before and after cold-water immersion of lower limbs. Methods: Thirty-two male subjects participated in the study, being randomly divided into two groups: (A) control group, immersion in water at $24^{\circ} \mathrm{C}$ for 10 minutes, (B) intervention group, immersion in cold-water at $10^{\circ} \mathrm{C}$ for 10 minutes. The volunteers performed evaluation of balance through a baropodometer, then they ran straight and in zigzag for 100 meters, entered in cold-water immersion, ending with a new assessment. We analyzed physical performance throughout the time of race, the heart rate by means of a frequency counter, and balance through a baropodometer and stabilometry in bipedal support with their eyes open. Results: The physical performance was modified after immersion in cold-water in intragroup and intergroup analysis. The heart rate showed difference when comparing before and after cryotherapy, but no differences compared to control. The balance showed no alteration after cryotherapy nor in comparison with the control group. Conclusion: Cryotherapy impaired physical performance when the activity was performed immediately thereafter, therefore ice is not recommended when you want performance in sporting activities. Nevertheless, ice had no interference on heart rate and balance of indoor soccer players, which may not have been altered due to the time of immersion.

Keywords: evaluation, induced hypothermia, athletic performance, postural balance.

\section{RESUMEN}

Introducción: Crioterapia es la aplicación de diferentes modalidades de frio con temperatura de $0^{\circ} \mathrm{C}$ a $18,3^{\circ} \mathrm{C}$, pudiendo interferir en el rendimiento físico y en el equilibrio, dependien do de la capacidad del individuo de mantener la estabilización, direccionar padrones de movimientos, controlar la postura y posición articular. Objetivo: Evaluar el rendimiento físico, la frecuencia cardíaca y el equilibrio estático con ojos abiertos, en jugadores de fútbol sala, antes y después de crioinmersión en los miembros inferiores. Métodos: Treinta y dos individuos del género masculino participaron del estudio, distribuidos aleatoriamente en dos grupos: (A) grupo control, inmersión en agua a $24^{\circ} \mathrm{C}$ 
por 10 minutos; (B) grupo intervención, crioinmersión en agua con hielo a $10^{\circ} \mathrm{C}$ durante 10 minutos. Los voluntarios realizaban la evaluación del equilibrio en un baropodómetro, luego corrían en línea recta y en zigzag durante 100 metros, entraban en la crioinmersión, finalizando con una nueva evaluación. El rendimiento físico fue analizado a través de los tempos de carreras, la frecuencia cardíaca, por medio de un frecuencímetro cardiaco, y el equilibrio a través de baropodometría y estabilometría en apoyo bipodal con ojos abiertos. Resultados: El rendimiento físico fue modificado después de la crioinmersión, tanto cuando analizados intra como intergrupal. La frecuencia cardíaca presentó diferencia significativa al comparar-se antes e después de la crioterapia, pero sin diferencias en comparación al control. El equilibrio no fue alterado después de la crioterapia ni en comparación con el grupo control. Conclusión: La crioinmersión afectó negativamente el rendimiento físico, cuando la actividad fue realizada inmediatamente después de la misma, siendo de esta manera la crioinmersión no aconsejable cuando se desea incrementar el rendimiento en la actividad deportiva. Pero no presentó interferencia en la frecuencia cardíaca ni en el equilibrio en jugadores de fútbol sala, que pueden no haber sido alterados debido al tempo da crioinmersión.

Palabras clave: evaluación, hipotermia inducida, rendimiento atlético, equilibrio postural.

\section{INTRODUÇÃO}

O termo crioterapia é utilizado para descrever a utilização do frio como tratamento que utiliza variação de temperatura de $0^{\circ} \mathrm{C}$ a $18,3^{\circ} \mathrm{C}$. O frio é um estado caracterizado pela diminuição de movimento molecular. Durante a crioterapia, o calor é retirado do corpo e absorvido, obtendo-se uma série de respostas locais e sistêmicas. A magnitude desses efeitos está relacionada com a temperatura da modalidade, a duração do tratamento e a superfície exposta ao tratamento ${ }^{1,2}$.

Os efeitos do frio começam pela analgesia, dada após a temperatura alcançar $13,6^{\circ} \mathrm{C}$, seguida pelo decréscimo da condução nervosa que reduz $10 \%$ a $12,5^{\circ} \mathrm{C}$, auxiliando na analgesia. A cada grau Celsius diminuídos, há uma diminuição de 2,4 m na velocidade da condução nervosa, reduzida por 30 min após ter sido retirado a modalidade fria. Além disso, o frio diminui o impulso nervoso na fenda sináptica, proporcionando como consequência um decréscimo da liberação de neurotransmissor (acetilcolina). Por fim, entre $10^{\circ} \mathrm{Ce} 11^{\circ} \mathrm{C}$ acontece uma diminuição de $50 \%$ da atividade metabólica enzimática ${ }^{3-6}$.

A redução da temperatura local pode interferir nas informações sensitivas e motoras. Um retardo na velocidade de propagação do impulso nervoso das fibras aferentes sensoriais, e uma redução da excitabilidade dos mecanorreceptores musculares implicarão alterações tanto ao nível da percepção de estímulos, como da ativação das unidades motoras, o que diminui a coordenação de padrões de movimento e estabilidade ${ }^{7-9}$.

As informações sensório-motoras têm grande relevância na percepção da estática e do movimento articular, oriundas dos receptores musculares, tendíneos, receptores localizados nos ligamentos, cápsulas articulares, meniscos e tecidos cutâneos. Assim, uma redução desta, imposta por programas de crioterapia, poderá diminuir a coordenação neuromuscular, equilíbrio e ser apresentada através de alterações da noção da posição articular, deteç̧ão limiar do movimento passivo e reprodução de força, aumentando, deste modo, a probabilidade de lesão e/ou uma redução do desempenho desportivo ${ }^{10-12}$.

O equilíbrio é um processo complexo que depende da integração da visão, da sensação vestibular e periférica, dos comandos centrais e respostas neuromusculares e, particularmente, da força muscular e do tempo de reação. O desempenho de habilidades motoras complexas, como os gestos esportivos, requer grande domínio sobre o equilíbrio. Um adequado controle do equilíbrio se reflete em sinergias musculares apropriadas, produzindo respostas motoras efetivas, as quais minimizam e restauram o equilíbrio. $O$ treinamento e a experiência tornam mais eficientes à ação motora e, por consequência, o desempenho físico e o equilíbrio em atletas. Aprender e treinar um esporte por um período longo de tempo parece melhorar o desempenho do atleta. Estudos prévios demonstram que o controle sobre o equilíbrio é um fator importante para o seu desempenho $0^{11-14}$.

O futsal no estado de Sergipe, Brasil, vem crescendo a cada ano, se popularizando e tornando-se cada vez mais em um esporte de alto rendimento, de grande impacto, devido aos jogadores não terem mais posição fixa e sua intensa movimentação, com acelerações e desacelerações bruscas. Os atletas de futsal estão propensos a diversas lesões osteomioarticulares e a utilização da crio é bastante frequente na recuperação dessas lesões. Entretanto, encontram-se poucos artigos relacionados com a crio pré-atividade ${ }^{15,16}$.

O objetivo deste estudo foi avaliar o desempenho físico, a frequência cardíaca e o equilíbrio estático com olhos abertos, em atletas de futsal, antes e depois da crioimersão nos membros inferiores.

\section{MÉTODOS}

Todos os procedimentos propostos pelos pesquisadores foram submetidos e aprovados ao Comitê de Ética da instituição, com CAAE no 0299.0.107.000-11. Antes das avaliações, os sujeitos eram solicitados a assinar o Termo de Consentimento Livre e Esclarecido, caso concordassem em participar da pesquisa.

O exame para a avaliação do equilíbrio estático antes e depois da imersão e da crioimersão foi realizado por meio do baropodômetro marca Arkipelago (modelo único, Arkipelago, São Paulo, Brasil), que consiste em um sistema de plataforma interligada ao computador, para mensurar as pressões exercidas pelos pés através do Sistema de Aquisição Computadorizada das Pressões (ACP) - Buratto Advance Tecnollogy (BAT-AM3,Paris,França) e de estabilômetro acoplado ao baropodômetro, que objetiva visualizar o centro de oscilação da pressão dos membros inferiores e do corpo (COP). A plataforma utilizada (dimensões $700 \mathrm{X}$ 600 mm, com 2544 sensores), foi conectada ao computador HP (São Paulo, Brasil), processador 6.2GHV, 4GB de memória RAM, disco rígido 500GB e software FOOTWORK (AM3, Paris, França), para analisar os dados captados. Para esses registros ortostáticos, foi utilizada a frequência de aquisição dos dados de $5 \mathrm{HZ}$ que perfaz cem leituras a cada avaliação.

Para a realização da imersão e crioimersão foram utilizados dois tonéis de plástico de 100 litros cada, um para imersão e o outro para crioimersão. Os dois tonéis tinham dimensões iguais, altura de $66 \mathrm{~cm}$ e diâmetro de $46 \mathrm{~cm}$. A água que foi usada para a imersão e a crioimersão foi do próprio abastecimento e o gelo para a crioimersão foi fabricado com a máquina de fazer gelo (Filco, Suggar NE 1011PR, Belo Horizonte, Brasil). O controle da temperatura da água da imersão e da crioimersão foi feito com termômetro digital (Alla, termo-higrômetro digital 760, 
São Paulo, Brasil). A corrida em linha reta e em ziguezague era realizada numa pista de atletismo, a uma distância de 100 m, previamente mensurada e marcada através de uma trena de 40 m (marca Starfer, classe III p1005, São Paulo, Brasil). O tempo foi medido, por um técnico em atletismo com 3 anos de experiência em provas de velocidade, utilizando-se um cronômetro (Casio, modelo HS-80, São Paulo, Brasil).

A frequência cardíaca era monitorada através de um frequencímetro (marca Polar modelo MFC FT1, Oulu, Finlândia) durante todo o processo e medida antes e depois das corridas. Foram marcados os tempos dos 100 m, por três avaliadores, e extraída uma média dos tempos.

Os participantes desta pesquisa foram distribuídos de forma aleatória em dois grupos: grupo A e grupo B, controle e intervenção, respectivamente.

Todos os participantes da pesquisa receberam orientações sobre a realização da avaliação do equilíbrio estático, feita através do baropodômetro, da avaliação do desempenho físico com circuito em linha reta e ziguezague de cem metros, assim como dos procedimentos da imersão e da crioimersão dos membros inferiores que o grupo controle e o grupo experimental realizaram, respectivamente, antes que fossem executados.

O estudo foi realizado em três etapas:

Na primeira etapa, os dois grupos responderam à ficha de avaliação e foram coletados os sinais vitais. Em seguida, foi avaliado o equilíbrio estático de olhos abertos, em apoio bipodal, antes e imediatamente após a imersão (controle) e a crioimersão (intervenção) através do baropodômetro. Realizou-se o teste de velocidade em linha reta e o de velocidade em ziguezague de $100 \mathrm{~m}$, onde foram marcados os tempo dos 100 m, por três avaliadores, e extraído uma média dos tempos. Antes e após cada tipo de corrida foi coletada também a frequência cardíaca.

Antes da avaliação, foi realizado o teste de alergia "Cubo de gelo", que consiste em colocar um cubo de gelo sobre a pele do indivíduo por 5 min; observa-se a resposta apresentada no local da aplicação após $10 \mathrm{~min}$.

A avaliação estabilométrica bipodálica foi feita com os indivíduos apoiados sobre a plataforma com apoio dos dois pés, sempre descalços, e braços relaxados ao longo do corpo. Os sujeitos foram instruídos a permanecerem na posição em pé, com os pés ligeiramente separados por uma largura confortável (de acordo com a largura dos ombros). 0 procedimento foi repetido três vezes. Em todos os casos o aparelho foi calibrado durante 10 seg para a execução dos procedimentos.

Na etapa dois, após a realização do teste de velocidade em linha reta e em ziguezague de 100 m, os indivíduos do grupo A (controle) foram submetidos à imersão dos membros inferiores até a altura do terço médio do fêmur, com água à $24^{\circ} \mathrm{C}$ durante $10 \mathrm{~min}$. A crioimersão dos membros inferiores até a altura do terço médio do fêmur que os indivíduos do grupo B (experimental) foram submetidos foi realizada com água adicionada ao gelo até atingir $10^{\circ} \mathrm{C}$ durante, $10 \mathrm{~min}$. Durante a aplicação da crioimersão, a temperatura foi acompanhada com o auxílio de um termômetro clínico digital, a fim de manterem-se as temperaturas preconizadas, através da retirada ou da colocação de cubos de gelo. Tanto os participantes do grupo A quanto do grupo B tiveram auxílio de uma escada de dois degraus para imergirem os membros inferiores no tambor de plástico.

Na última etapa, imediatamente após a imersão ou crioimersão. Foi realizada a corrida de $100 \mathrm{~m}$ em linha reta e ziguezague e uma nova coleta da frequência cardíaca antes e após cada tipo de corrida. Depois, avaliou-se com o baropodômetro o equilíbrio estático dos indivíduos dos grupos A e B, conforme descrito anteriormente.

Os dados foram analisados através do programa estatístico BioEstat versão 5.0. (Belém, Pará - Brasil) A partir deste, foram utilizados mediana ( \pm erro padrão da média) para análise descritiva dos dados, os testes de Shapiro-wilk para verificar distribuição de probabilidade das variáveis quantitativas; teste de Wilcoxon (signed-rank) para análise comparativa dos dados, utilizado para analisar variáveis não pareadas e dependentes (considerando $\mathrm{p} \leq$ 0,05), o teste de Mann-whitney (Wilcoxon Rank-sum Test) para variáveis não pareadas e independentes.

\section{RESULTADOS}

Participaram desse estudo 35 voluntários, sendo que três foram excluídos (um por desmaiar na crioimersão, um não suportou ficar os 10 min na crioimersão e o outro porque no teste físico teve uma lesão muscular).

O desempenho físico avaliado através do tempo da corrida em linha reta 100 m e em ziguezague 100 m apresentou diferença significativa, quando avaliado através de suas medianas ( \pm erro padrão). Verificou-se a diminuição no desempenho, quando analisado a relação intergrupo da corrida em linha reta de $100 \mathrm{~m}$ após a crioimersão do grupo intervenção, que foi de 23,21s ( $\pm 0,70)$; já no grupo controle, o valor foi de 20,11s $( \pm 0,26)$, com $p<0,0001$. Com relação à corrida em ziguezague de $100 \mathrm{~m}$ após a crioimersão, o grupo intervenção atingiu a marca de $38,50 \mathrm{~s}( \pm 0,42)$, enquanto que grupo controle atingiu $35,87 \mathrm{~s}$ $( \pm 0,62)$ com $p<0,02$, sendo estes valores de $p$ obtidos através do teste de Mann-whitney (figura 1).

Nos dois tipos de corrida, antes da crioimersão, não houve diferença significativa. Na análise intragrupos, observou-se uma queda no desempenho, quando analisado o tempo da corrida em linha reta de 100 m do grupo intervenção após a crioimersão, que foi de 23,21s $( \pm 0,70)$, comparado a antes da crio que foi de $19,91 \mathrm{~s}( \pm 0,23)$, com $p$ $<0,0007$. Uma diferença foi observada na corrida de 100 metros em ziguezague do grupo intervenção após a crioimersão, com mediana de 38,50 s $( \pm 0,42)$, enquanto que antes da crio o valor obtido foi 35,79s $( \pm 0,54)$ com $p<0,0007$. Já o grupo controle não apresentou diferença significativa. Todas as análises de p foram obtidas através do teste de Wilcoxon. Estes valores estão representados na tabela 1.

A frequência cardíaca ( $F C$ ) foi avaliada nos seguintes momentos: antes da crioimersão ou imersão (FC1), após a corrida de 100 m linha reta antes da crio ou imersão (FC2), após a corrida de 100 m em ziguezague antes da crio ou imersão (FC3), após a crio ou imersão (FC4), após a corrida em linha reta de 100 m após a crio ou imersão (FC5) e após a corrida em ziguezague de 100 m após a crio ou imersão (FC6).

Na análise intergrupo, apenas se apresentou diferença significativa na FC após a corrida linha reta de $100 \mathrm{~m}$ antes da crio, onde o grupo intervenção atingiu 148bpm $( \pm 2,05)$ enquanto o grupo controle 141 bpm $( \pm 2,64)$ com p igual a $<0,0001$. Os outros cruzamentos intergrupo não apresentaram diferença significativa, como representado na figura 2.

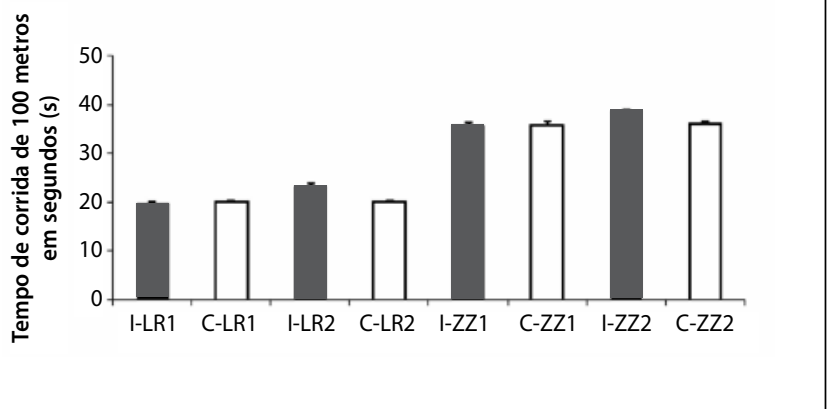

Figura 1. Análise intergrupo do desempenho durante a corrida de $100 \mathrm{~m}$. 
Na análise intragrupos, apresentou-se aumento da frequência cardíaca com diferença significativa quando comparadas as seguintes variáveis: Frequência inicial grupo intervenção após a crio 98bpm $( \pm 3,80)$ e antes da crio 83bpm $( \pm 1,98)$ com p igual a 0,001; grupo controle após a imersão 97bpm $( \pm 2,60)$ e antes da imersão 80bpm $( \pm 2,33)$, com p igual a 0,0036; frequência após a corrida em linha reta de $100 \mathrm{~m}$, onde o grupo intervenção não apresentou diferença significativa; o grupo controle apresentou após a imersão 149bpm $( \pm 3,28)$ e antes da imersão 141 bpm $( \pm 2,49)$ com p igual a 0,0028; já a frequência após a corrida de $100 \mathrm{~m}$ em ziguezague no grupo intervenção foi após a crio $167 \mathrm{bpm}( \pm 2,41)$ e antes da crio $172 \mathrm{bpm}( \pm 1,81)$ com p igual a 0,0012 ; o grupo controle após a imersão obteve a média de $175 \mathrm{bpm}( \pm 3,03)$ e antes da imersão 168bpm $( \pm 3,41)$ com p igual a 0,0036 . Porém com exceção da variável linha reta do grupo intervenção, todas as outras apresentaram diferença significativa (tabela 2).

O Centro de Oscilação de Pressões (COP) não apresentou diferença significativa na análise intergrupo ( $p<0,37)$, e nem na análise intragrupo $(p<0,19)$, através da análise das medianas (erro padrão) das variáveis do Grupo intervenção COP antes da crioimersão e após a crioimersão; Grupo controle antes da crioimersão e após a crioimersão (figura 3).

A pressão média apresentou diferença significativa apenas na análise intragrupos da variável pressão média do pé esquerdo do grupo controle antes da crioimersão, que foi de $0,31 \pm 0,01 \mathrm{Kgf} / \mathrm{cm}^{2}$ e depois da crioimersão que foi $0,25 \pm 0,01 \mathrm{Kgf} / \mathrm{cm}^{2} \mathrm{com} p$ igual a 0,0092. As variáveis intragrupo $p<0,44$ e intergrupo $p<0,86$ não apresentaram diferença significativa (figura 4).

Tabela 1. Análise intragrupo do desempenho durante a corrida de $100 \mathrm{~m}$.

\begin{tabular}{c|c|c|c|c}
\hline & Antes da crio & Após a crio & Aumentou \% & Valor de "P" \\
\hline $\begin{array}{c}\text { Grupo intervenção } \\
\text { linha reta }\end{array}$ & $19,91( \pm 0,23)$ & $23,21( \pm 0,70)$ & 16,57 & ${ }^{*} 0,0007$ \\
\hline $\begin{array}{c}\text { Grupo controle } \\
\text { linha reta }\end{array}$ & $20,06( \pm 0,28)$ & $20,11( \pm 0,26)$ & 0,24 & 0,5540 \\
\hline $\begin{array}{c}\text { Grupo intervenção } \\
\text { ziguezague }\end{array}$ & $35,79( \pm 0,54)$ & $38,5( \pm 0,42)$ & 7,57 & ${ }^{*} 0,0007$ \\
\hline $\begin{array}{c}\text { Grupo controle } \\
\text { ziguezague }\end{array}$ & $35,77( \pm 0,65)$ & $35,87( \pm 0,62)$ & 0,27 & 0,6791 \\
\hline
\end{tabular}

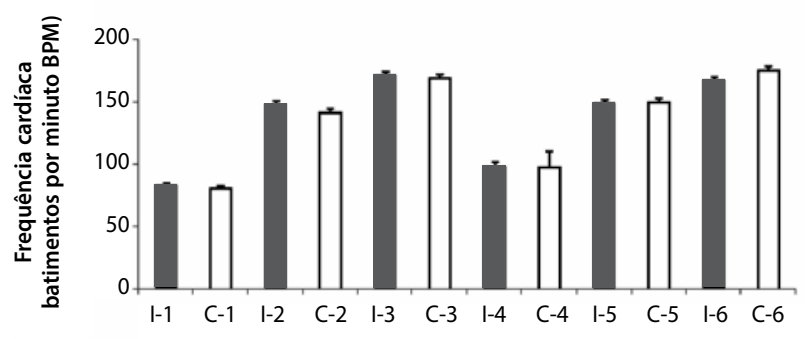

Figura 2. Análise intergrupo da frequência cardíaca.

Tabela 2. Análise intragrupo da frequência cardiaca antes e após a crioterapia.

\begin{tabular}{c|c|c|c}
\hline & Antes da crio & Após a crio & Valor de “P” \\
\hline Grupo intervenção FCl & $83( \pm 1,98)$ & $98( \pm 3,80)$ & ${ }^{*} 0,0010$ \\
\hline Grupo controle FCl & $80( \pm 2,33)$ & $97( \pm 2,60)$ & ${ }^{*} 0,0036$ \\
\hline Grupo intervenção FCLR & $148( \pm 2,05)$ & $149( \pm 2,34)$ & 0,9001 \\
\hline Grupo controle FCLR & $141( \pm 2,49)$ & $149( \pm 3,28)$ & ${ }^{*} 0,0028$ \\
\hline Grupo intervenção FCZZ & $172( \pm 1,81)$ & $167( \pm 2,41)$ & ${ }^{*} 0,0012$ \\
\hline Grupo controle FCZZ & $168( \pm 3,41)$ & $175( \pm 3,03)$ & ${ }^{*} 0,0036$ \\
\hline
\end{tabular}

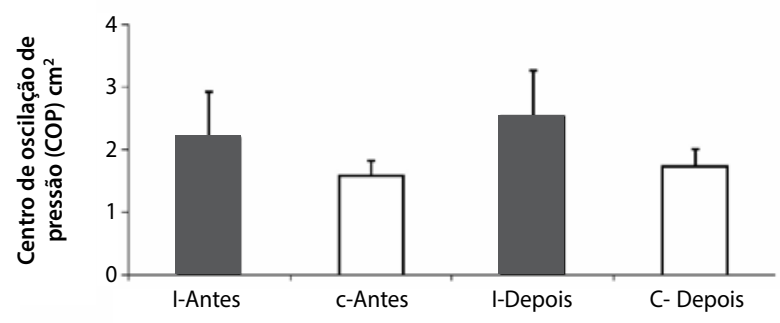

Figura 3. Análise intergrupo e intragrupo do centro de oscilação de pressão.

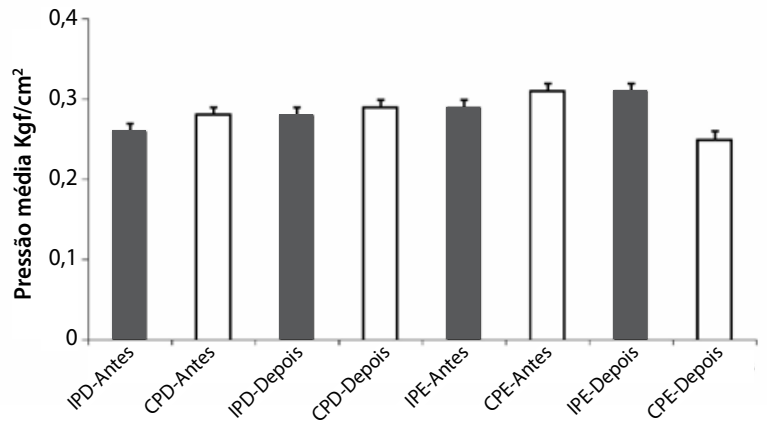

Figura 4. Análise intergrupo e intragrupo da pressão média.

\section{DISCUSSÃO}

Nesse estudo analisou-se o desempenho físico, frequência cárdica e equilíbrio sob influência da crioimersão. Encontrou-se, porem, muitas dificuldades para confrontar os resultados obtidos, devido à escassez de artigos relacionados a este tema.

Na avaliação do desempenho físico em relação ao tempo da corrida em linha reta e ziguezague, as marcas alcançadas após a crioimersão foram piores do que as alcançadas antes; os atletas avaliados relataram queixas da sensação de que as pernas estavam pesadas após a crioimersão. Isto pode ser explicado pelo resfriamento, que diminui a sensibilidade do fuso, justificando a diminuição da percepção da capacidade contrátil do músculo. Pela menor velocidade de condução nervosa do nervo motor, a força muscular é prejudicada, além de sua potência, aumentando-se o risco de lesões, uma das contraindicações da crioterapia antes da atividade física ${ }^{6,17,18}$. Mais recentemente, num estudo de Levels et al., os autores concluíram que o resfriamento do couro cabeludo associado a ingesta de bebidas geladas, antes de provas de ciclismo de $15 \mathrm{Km}$, foi mais benéfico para o tempo dos atletas, ainda que sua performance em geral não tivesse sido modificada, como ocorrido no presente estudo.

Em estudo realizado por Ruiz et al. ${ }^{20}$, foi encontrada a redução da força muscular do quadríceps após a utilização do gelo, bem como Duarte e Macedo ${ }^{21}$ também encontraram este mesmo resultado. Kinzey et al. ${ }^{22}$ sugere que a força muscular fica prejudicada após o uso da crioterapia, devido à redução da velocidade de condução do nervo motor. Pereira et al. ${ }^{23}$ afirmaram que a força muscular é reduzida após o resfriamento, sendo assim, o atleta ficaria exposto mais facilmente a lesões. Contrariando esses argumentos, Evans et al. ${ }^{24}$ relataram que a crioterapia não interfere no desempenho funcional de atletas. Tremblay et al. ${ }^{25}$ concorda que a crioimersão não altera o desempenho motor, porém altera a força e a flexibilidade muscular. Rubley et al. ${ }^{26}$ afirma que a produção de força não é afetada pela crioterapia.

Como esperado, a frequência cardíaca foi aumentando com o teste físico nos dois grupos, observando-se o mesmo grupo. Isto pode ser 
justificado pelo esforço realizado para completar a corrida, o que propicia um esforço maior também sobre o coração, porém era esperado que houvesse diferença estatística significante, com o valor das medianas do grupo intervenção mais altas quando comparadas ao grupo controle, porém somente uma apresentou tal resultado, tendo em vista que o coração iria trabalhar para que o corpo não sentisse tanto os efeitos do frio, e, com isso, sua frequência aumentaria, o que não ocorreu. $O$ tempo de crioimersão, de apenas 10 min, pode não ter sido suficiente para causar grandes alterações sistêmicas, que pudessem ter sido refletidas no trabalho cardíaco. Este pode ter sido o motivo pelo o qual o grupo intervenção não apresentou maior variação da frequência cardíaca, comparado ao grupo controle. ${ }^{27}$

Segundo Alonso et al. ${ }^{27}$, durante o exercício progressivo, a frequência cardíaca aumenta concomitantemente ao aumento no consumo de oxigênio e ao aumento de potência relativa. Essa relação entre a FC e o consumo de oxigênio demonstra que o comportamento da FC durante o exercício depende, em grande parte, da demanda metabólica na musculatura ativa, expressando um aumento da FC, com o aumento da intensidade do exercício. O pré-resfriamento, porém, induziria à diminuição do estresse cardíaco, bem como à redução do gasto metabólico geral e supressão dos sinais centrais de inibição ${ }^{28}$.

A influência do gelo no equilíbrio mostrou que a crioimersão não apresentou diferença estatística, tanto na análise intragrupo como intergrupo, do centro de oscilação de pressão (COP) e nem na análise das pressões médias do pé direito e do pé esquerdo, com exceção da análise pé esquerdo. Isso se deu no equilíbrio estático, o que pode gerar a dúvida de que, no equilíbrio dinâmico, este comportamento seja diferente. Como o equilíbrio é controlado por diversos fatores, apesar de o resfriamento interferir na propriocepção, os outros fatores de controle passam a suprir o déficit da propriocepção e mantêm o equilíbrio. A função proprioceptiva é importante para o desempenho esportivo, por isso a crioimersão pode aumentar os riscos de lesões ${ }^{6,13,24}$.

A propriocepção é definida como uma variação especializada da modalidade sensorial, tendo um papel fundamental na percepção da estática e do movimento articular. Hopper et al. ${ }^{29}$ observaram que a crioterapia não diminui o senso de posição articular, e com isso, exercícios após a crioterapia não ofereceriam riscos ao equilíbrio. Para Wassinger et al. ${ }^{30}$, a propriocepção é diminuída após a crioterapia e os fisioterapeutas e treinadores desportivos devem estar atentos aos déficits neuromotores causados pelo resfriamento, pois esta modalidade é comumente utilizada após lesões agudas e durante o processo de reabilitação, quando sobrecargas são impostas às estruturas do sistema musculoesquelético envolvidas após crio.

\section{CONCLUSÃO}

Infere-se que a crioimersão pode prejudicar o desempenho físico, se a atividade for imediatamente após a crio, sendo desta forma o gelo não aconselhável quando se deseja desempenho na atividade desportiva. Em relação à frequência cardíaca na análise intragrupo apresentou diferença significativa, mas quando comparado o grupo controle e intervenção não apresentou diferença. Sendo assim, a crioimersão não resultou em diferença quando da sua utilização ou não, o que pode ter sido devido ao tempo da crio. Por fim, o equilíbrio estático também não foi afetado após a utilização da crioimersão, muito provavelmente porque o tempo de crioimersão foi de apenas 10 min e, apesar da crio prejudicar a propriocepção, os outros componentes que controlam o equilíbrio podem suprir a deficiência na propriocepção.

Todos os autores declararam não haver qualquer potencial conflito de interesses referente a este artigo.

\section{REFERÊNCIAS}

1. Guirro R, Adib C, Maximo C. Os efeitos fisiológicos da crioterapia: uma revisão. Rev Fisioter Univ São Paulo.1999;6(2):164-70.

2. Umphred DA. Reabilitação neurológica. 4a ed. São Paulo: Manole; 2004,

3. Cardoso CS, Santos VBC, Macedo CSG. Aplicação de crioimersão em atletas durante a taça Brasil de futsal feminino. Rev Ciênc Saúde. 2009;Número Especial:58. Disponível em : http://revistaseletronicas. pucrs.br/ojs/index.php/faenfi/article/view/6138/4429

4. Prentice WE. Modalidades terapêuticas em medicina esportiva. São Paulo: Manole; 2002.

5. Low J, Reed A. Eletroterapia explicada: princípios e prática. São Paulo: Manole; 2001.

6. Knight KL. Crioterapia no tratamento das lesões esportivas. São Paulo: Manole; 2000.

7. Felice TD, Ishizuka ROR, Amarilha JD. Eletroestimulação e crioterapia para espasticidade em pacientes acometidos por acidente vascular cerebral. Rev Neurocienc. 2011;19(1):77-84.

8. Atnip BL, McCrory JL. The effect of cryotherapy on three dimensional ankle kinematics during a sidestep cutting maneuver. J Sports Sci Med. 2004;3(2):83-90.

9. Dover G, Powers ME. Reliability of joint position sense and force-reproduction measures during internal and external rotation of the shoulder. J Athl Train. 2003;38(4):304-310

10. Borsa PA, Lephart SM, Kocher SM, Lephart SP. Funcional assessment and rehabilitation of shoulder proprioceptation for glenoumeral instability. J Sport Rehabil; 1994;3:84-104.

11. Hubbard TJ, Aronson SL, Denegar CR. Does Cryotherapy Hasten Return to Participation? A Systematic Review. J Athl Train. 2004;39(1):88-94.

12. Hayes K, Callanan M, Walton J, Paxinos A, Murrell GA. Shoulder instability: management and rehabilitation. J Orthop Sports Phys Ther. 2002;32(10):497-509.

13. Leporace G, Metsavaht L, Sposito MMM. Importância do treinamento da propriocepção e do controle motor na reabilitação após lesões músculo-esqueléticas. Acta Fisiátr. 2009;16(3):126-31.

14. Silva A, Almeida GJM, Cassilhas RC, Cohen M, Peccin MS, Tufik S, et al. Equilíbrio, coordenação e agilidade de idosos submetidos à prática de exercícios físicos resistidos. Rev Bras Med Esporte. 2008;14(2):88-93.

15. Souza TC, Medeiros C, Silva LA, Silveira TC, Silveira PC, Pinho CA, et al. Avaliação sérica de danos musculares e oxidativos em atletas após partida de futsal. Rev Bras Cineantropom Desempenho Hum. 2010;12(4):269-74.

16. Cyrino ES, Altimari LR, Okano AH, Coelho CF. Efeitos do treinamento de futsal sobre a composição corporal e o desempenho motor de jovens atletas. Rev Bras Ciên Mov. 2002;10(1):41-6.
17. Rodrigues A. Crioterapia: fisiologia e técnicas terapêuticas. São José do Rio Preto: Cefespar; 1995.

18. Pinheiro FB. Crioterapia. Estudo do Uso da Crioterapia na Fisioterapia e sua comprovação científica. In: Anais com Congresso da Universidade do grande ABC, 03-05/03/2008. São Paulo: Artmed, 2000, p. 35-36.

19. Levels K.; Teunissen L. P.J; de Haan A.; de Koning J.J; van Os B.; Daanen H. A.M... Effect of warm-up and precooling on pacing during a $15-\mathrm{km}$ cycling time trial in the heat. Int J Sports Physiol Perform. 2013 May;8(3):307-11.

20. Ruiz DH, Myrer JW, Durrant E, Fellingham GW. Cryotherapy and sequential exercise bouts following cryotherapy on concentric and eccentric strength in the quadriceps. J Athl Train. 1993;28(4):320-3.

21. Duarte R, Macedo R. Efeito do gelo no momento máximo de força durante o movimento concêntrico de extensão do joelho. EssFisiOnline. 2005;1(3):21-37. Disponível em: http://www.fisionline.ips.pt/ media/essfisionline/vol1n3.pdf

22. Kinzey SJ, Cordova ML, Gallen KJ, Smith JC, Moore JB. The effects of cryotherapy on ground reaction forces produced during a functional task. J Sport Rehabil. 2000;9(1):3-14.

23. Pereira LG, Pereira R, Sampaio-Jorge F, Magini M. Avaliação da força muscular do tibial anterior após aplicação local de crioterapia. Revista UNIVAP. 2007;online:1917-20.

24. Evans TA, Ingersoll C, Knight KL, Worrell T. Agility following the application of cold therapy. J Athl Train. 1995;30(3):231-4.

25. Tremblay F, Estephan L, Legendre M, Sulpher S. Influence of local cooling on proprioceptive acuity in the quadriceps muscle. J Athl Train. 2001;36(2):119-23.

26. Rubley MD, Denegar CR, Buckley WE, Newell KM. Cryotherapy, sensation, and isometric-force variability. J Athl Train. 2003;38(2):113-9.

27. Alonso DO, Forjaz LM, Rezende LO, Braga AMFW, Baretto ACP, Negrão CE, et al. Comportamento da freqüência cardíaca e da sua variabilidade durante as diferentes fases do exercício físico progressivo máximo. Arq Bras Cardiol. 1998;71(6):787-92.

28. Wegmann, M.; Faude, O.; Pofpendieck, W.; Hecksteden, A.; Fröhlich, M.; Mey, T.. Pre-Cooling and Sports Performance: A Meta-Analytical Review. Sports Med 2012: 42 (7): 545-564.

29. Hopper D, Whittington D, Davies J. Does ice immersion influence ankle joint position sense? Physiothe Res Int. 1997;2(4):223-36

30. Wassinger, C. A.; Myers, J. B.; Gatti, J. M.; Conley, K. M.; Lephart, S. M. Proprioception and Throwing Accuracy in the Dominant Shoulder After Cryotherapy. Journal of Athletic Training; vol. 42, n.1, p.84-89, 2007. 